\title{
Plasmodesmata during development: re-examination of the importance of primary, secondary, and branched plasmodesmata structure versus function
}

\author{
Tessa M. Burch-Smith • Solomon Stonebloom • \\ Min Xu $\cdot$ Patricia C. Zambryski
}

Received: 6 August 2010 /Accepted: 16 October 2010 /Published online: 21 December 2010

(C) The Author(s) 2010. This article is published with open access at Springerlink.com

\begin{abstract}
Plasmodesmata (PD) structure and function vary temporally and spatially during all stages of plant development. PD that originate during, or post, cell division are designated as primary or secondary according to classical terminology. PD structure may be simple, twinned, or branched. Studies of PD during leaf, root, and embryo development have lead to the generalization that cells in less mature tissues contain predominantly simple PD. New quantitative analyses reveal that twinned and branched PD also occur in immature tissues. New data also highlight the versatility of viral movement proteins as tags for labeling PD in immature tissues as well as PD in mature tissues. A summary of the formation and function of primary, secondary, and branched PD during leaf, trichome, embryo, apical meristem, vascular cambium, and root development underscores the remarkable and indispensible plant-specific intercellular communication system that is mediated by PD.
\end{abstract}

Keywords Plasmodesmata $\cdot$ TMV-MP. Plant development . Primary and secondary plasmodesmata. Intercellular movement

\section{Introduction}

Coordinated growth, development, response to the environment, and defense in multi-cellular organisms require

Handling Editor: Alexander Schulz

T. M. Burch-Smith · S. Stonebloom • M. Xu •

P. C. Zambryski $(\square)$

Department of Plant and Microbial Biology,

University of California,

Berkeley, CA 94720, USA

e-mail: zambrysk@berkeley.edu the exchange of information between cells. In plants, cellto-cell communication must occur despite the presence of cellulosic walls surrounding each individual cell. This difficulty is circumvented by the use of cell wall spanning, membrane-lined, cytoplasmic channels known as plasmodesmata (PD). PD connect nearly all the cells of a plant, allowing the transport of nutrients, signaling molecules, and developmentally important macromolecules such as transcription factors and RNA (reviewed in (Cilia and Jackson 2004; Lucas and Lee 2004; Kim and Zambryski 2005)).

One of the most critical and unique functions of PD during plant development is the formation of symplastic domains. During embryonic development, all cells share common cytoplasm due to PD interconnections and thus form one single symplastic unit allowing transport of small fluorescent tracers and green fluorescent protein (GFP) (McLean et al. 1997; Kim et al. 2005a). As growth and further differentiation occurs, individual cells or groups of cells become isolated from their immediately neighboring cells by the loss of functional PD. Such subsets of cells form distinct symplastic domains where cells within the domain communicate with each other via PD, while cells at the borders of the domains restrict intercellular transport between domains.

That PD have essential roles in plant growth and development is underscored by the lethal phenotypes of mutants exhibiting altered PD or plasmodesmal transport (Kim et al. 2002a; Kobayashi et al. 2007; Benitez-Alfonso et al. 2009, Stonebloom et al. 2009). In this review, we discuss the formation, structure, and distribution of PD during development. We focus on cell-to-cell movement and not long distance movement via the vascular system. There is a vast PD literature and wherever possible, we try to give only the most recent relevant references. For the reader interested in additional references and details, we 
suggest a book entitled "Plasmodesmata" (Oparka 2005). Other reviews are mentioned in specific subsections. We sincerely apologize if we have missed a relevant publication due to our unawares. As we pay special attention to PD nomenclature, we begin here.

PD nomenclature, structure, and function during development

Primary and secondary PD have been described and defined in a comprehensive review by Ehlers and Kollmann (2001); primary PD originate during cell division, and secondary PD refer to all PD that originate post cytokinesis. Besides differing origins, PD have numerous structures, from simple, to branched, to highly branched with central cavities (Fig. 1). The least complex branched PD are Y-, $\mathrm{V}-, \mathrm{X}-$, or H-shaped. Simple PD are most often found alone, but they also occur in pairs, called twinned PD, PD not more than 100-nm apart (Faulkner et al. 2008). Y-, V-, X-,

a

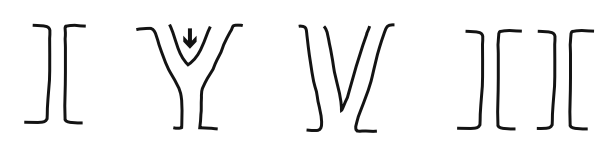

b

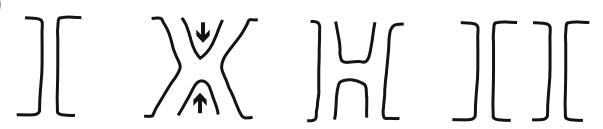

C
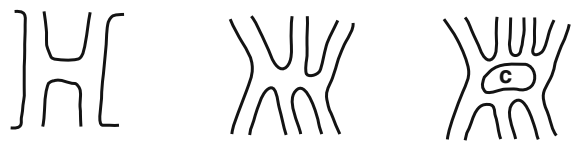

d

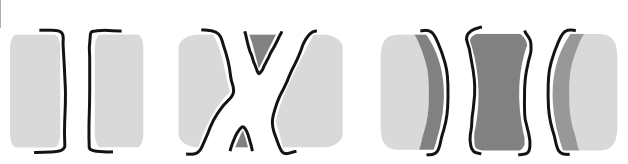

Fig. 1 PD structures and how they form. a, b Present different intermediate PD structures that may form during the creation of twinned PD by fission of a simple PD. a Initiation of PD doubling at one site that results in Y- and V-shaped intermediates. b Initiation of PD doubling at two sites that results in $\mathrm{X}$ - and H-shaped intermediates. c How an H-shaped PD may become further branched and ultimately contain a central cavity (c). d A mechanistic model for how twinned PD or branched PD arise that derives from the laying down of new cell wall material (dark grey shading) as the cell wall (light grey shading) expands laterally. New cell wall material may initiate the branching of PD and then ultimately separate the two newly formed PD channels. While the cell wall is not drawn in panels $\mathbf{a}, \mathbf{b}$, and $\mathbf{c}$, cell wall expansion likely also drives the formation of the Y-, V-, X-, and H-shaped PD, twinned PD, and multiple-branched PD. Note the PD channels diagrammed in all panels represent the cell membrane bound channel without the central ER-derived desmotubule. The model drawn in D is a simplification of a similar model in Ehlers and van Bel (2010) or H-shaped PD may represent intermediates in fission of simple PD to form twinned PD. Figure 1a diagrams twinned PD formation from a single initiation point that then results in PD doubling via Y- to V-shaped intermediates. Figure $1 \mathrm{~b}$ presents a scenario that involves two opposite initiation sites forming an $\mathrm{X}$-shaped intermediate that then becomes $\mathrm{H}$-shaped prior to resolving into twinned $\mathrm{PD}$. Figure 1c diagrams that complex branched PD with multiple channels and central cavities that may arise from $\mathrm{H}$-shaped branched PD.

Here, we suggest that $\mathrm{H}$-shaped PD represent intermediates in the formation of twinned PD (Fig. 1b) or they may represent modifications of simple PD that occur as cells and tissues mature to form complex multiply branched PD (Fig. 1c). Previously, numerous authors propose that $\mathrm{H}-$ shaped branched PD arise by fusion of twinned PD, possibly by an X-shaped intermediate (Ding and Lucas 1996; Ehlers and Kollmann 2001; Faulkner et al. 2008); such PD fusion implies H-shaped PD arise post-twin PD formation. However, here we put forward the hypothesis that PD fusion is an unlikely event as such fusions would imply that the cell wall regions between PD contract. Cell wall contraction may occur under certain circumstances such as during senescence, or environmental stress. However, during the bulk of plant development as plant cells grow, their cell walls expand, and PD are likely to double during cell wall expansion (Ehlers and van Bel 2010) (Fig. 1d) (discussed further below). Quantitative time course studies may resolve these conflicting views.

The frequency of simple to branched PD strongly correlates with plant development. In young, immature tissues like embryos (Burch-Smith and Zambryski 2010), sink leaves (Oparka et al. 1999), shoot (Ormenese et al. 2000), and root meristems (Duckett et al. 1994; Zhu et al. 1998a) PD are mostly morphologically simple, consisting of a single linear channel connecting adjacent cells. In mature tissues, PD are mostly branched, adopting more complex structures of multiple channels connected by central cavities (Ding et al. 1992). One of the best illustrations of PD structural alterations during development from immature to mature cells, is a quantitative study of simple versus branched PD during the sink to source transition in tobacco leaves (Oparka et al. 1999). These authors found that sink leaf cells contain over $90 \%$ simple PD. In contrast, source leaf cells contain predominantly highly branched PD with central cavities, and only approximately $20 \%$ simple PD.

The molecular details of how primary and secondary PD are formed are unknown. Models to date derive from descriptive ultrastructural studies that give only a static snapshot of PD structure and development following harsh chemical fixation of tissues. Nevertheless, we briefly summarize what is known. Primary PD always arise during 
cell division, when strands of endoplasmic reticulum (ER) become entrapped in the cell plate during Golgi-mediated deposition of new cell wall material; Golgi membranes then surround the ER strands to form the outer limits of the PD membrane (reviewed in Ehlers and Kollmann 2001).

An even bigger question is how secondary PD form? We now know that there are two types of secondary PD. Secondary PD that arise from simple PD to form twinned $\mathrm{PD}$, and secondary PD that arise entirely de novo in cell walls without pre-existing PD. We use the terms "twinned secondary PD" and "de novo secondary PD" to distinguish these two types of secondary PD. Figure 1d presents a simple model for how twinned secondary PD arise. In this model (from Ehlers and van Bel 2010), PD form as the cell wall loosens and expands horizontally and new cell wall material becomes inserted in between PD membranes. Cell wall expansion may also provoke the insertion of new PD channels as occurs during complex branched PD formation (Fig. 1c). Another model proposes new strands of ER become inserted nearby an existing PD localized ER strand, followed by insertion of new cell wall material (Faulkner et al. 2008). Ehlers and van Bel (2010) distinguish the role of the ER in these two models in PD fission, as "passive" and "active". We favor the passive model (cell wall deposition, not ER drives PD formation) presented in Fig. 1d as we never observe ER insertion into the cell wall during examination of thousands of PD that are forming in cells from developing immature tissues in embryos and sink leaves. It remains possible that ER insertion and/or entrapment may occur in other cells and tissues. Genetic, molecular, and advanced imaging approaches are clearly needed to resolve how twinned PD arise and subsequently develop.

How de novo secondary PD form is even more poorly understood. The critical question is how does the formation of a plasmodesma initiate? Does PD formation initiate in one cell and then progress through the cell wall to the adjacent cytoplasm? A more intricate and unlikely scenario would involve signaling of PD initiation at identical locations in adjacent cells resulting in PD fusion/formation in the central wall region. A plausible scenario involves localized thinning of the cell wall bringing the plasma membranes between two adjacent cells into proximity to allow PD formation to initiate in a focused region with subsequent vertical extension as new cell wall material is laid down. This intriguing possibility is supported by abundant data from graft unions, and fusions between cells growing in tissue culture (reviewed in Ehlers and Kollmann 2001).

However, few studies monitor de novo secondary PD formation during wild-type development. The most obvious cells to study de novo secondary PD formation are those undergoing cell wall elongation post cell division. Two such systems have been investigated in astonishing detail, the elongation zone of the developing root (Zhu et al. 1998a) and the development of secondary xylem and phloem (Ehlers and van Bel 2010). Both studies quantify PD in different cell types along apical, basal, and side cell walls, and are described in more detail in later sections of this review. Apical and basal root cell walls predominantly contain primary PD resulting from cell division in the horizontal plane, whereas elongating sidewalls contain de novo secondary PD. Other systems to study de novo secondary PD formation are those which characterize PD between cells that are unambiguously not clonally related, such as between the epidermal cell layer and the underlying mesophyll cells of the leaf (Burch-Smith and Zambryski 2010). Like primary PD, secondary PD can have various morphologies, from simple to branched (Ehlers and van Bel 2010; Burch-Smith and Zambryski 2010).

How then can primary PD be distinguished from secondary PD? In immature cell walls that are clearly formed as a result of cytokinesis, such as the mesophyll cells of a newly arising sink leaf or the transverse cell walls of a file of elongating root cells in the differentiation zone, PD can be classified most likely as primary. Twinned, Y-, or $\mathrm{H}$-shaped PD in rapidly dividing cells from embryos or sink tissues may represent different stages in the modification of primary PD to produce secondary twin or branched PD (Fig. 1). In contrast, in mature cell walls it is impossible to unequivocally assign the origin of PD as primary or secondary unless the lineage of the cell wall and the developmental history of the PD is known.

Finally, PD function is described by the types of molecules are transported by PD. We introduced the terms targeted and nontargeted PD movement (Crawford and Zambryski 2000). Proteins that interact with PD and induce their own efficient movement are designated "targeted" PD proteins. Targeted proteins, such as tobacco mosaic virus movement protein (TMV-MP) have a punctate appearance in cell walls and such puncta are coincident with PD. Nontargeted proteins, exemplified by exogenous proteins such as GFP, move cell to cell by diffusion and do not target/ localize to PD. Targeted movement is thought to be an active process compared with passive non-targeted movement. Whether a protein can move via PD or not is dictated by its cellular address (Crawford and Zambryski 2000); thus, proteins with ER retention or organelle targeting signal sequences do not move cell to cell as they are sequestered into different cellular compartments. Likewise, proteins that are anchored onto cellular structures such as the cytoskeleton do not move cell to cell via PD. Thus, only "soluble" nonsequestered cytoplasmic proteins can move cell to cell. Interestingly, the nuclear pore allows proteins to move into the cytoplasm and vice versa, so that nuclear proteins also can move cell to cell via PD (Crawford and Zambryski 2000). Notably, numerous transcription factors 
have been demonstrated to move to adjacent cells via PD (see below), and when expressed ectopically they move extensively. These latter data have led to the suggestion that transcription factors move cell to cell via diffusion, unless retained by their respective binding sites in active chromatin (Wu et al. 2003).

Two new results provoke renewed attention to PD nomenclature. Firstly, we (Kobayashi et al. 2007), and others (Benitez-Alfonso et al. 2009), previously assumed that embryos only contain simple unbranched PD; both reports mention the presence of branched PD specifically as a feature of the mutant phenotype. However, quantitative and statistically significant measurements of PD structures during embryogenesis now reveal that wild-type embryos contain 5-9\% twinned and branched PD between early and late torpedo stages of development (Burch-Smith and Zambryski 2010). Thus, the $9 \%$ branched PD reported by Benitez-Alfonso et al. (2009) is not significantly different from the frequency observed in wild-type embryos. While Kobayashi et al. (2007) noted 15\% branched PD in their mutant, they incorrectly reported no branched PD occur in wild-type tissues because insufficient numbers of PD were analyzed in wild-type tissues. Thus, to accurately determine the frequencies of different PD structures in particular cell types it is essential to analyze a statistically significant number (at least 500) of PD in both experimental and wildtype tissues.

Secondly, we have new results on the properties of the movement protein (MP) of tobacco mosaic virus (TMV). TMV-MP is an exceptionally valuable tool for PD research as TMV-MP labels PD, and TMV-MP can also manipulate PD aperture to increase intercellular movement (reviewed in (Epel 2009)). While TMV-MP previously has been used to identify PD as "secondary" (Ding et al. 1992), we present data (Fig. 2 below) showing that TMV-MP also extensively labels primary PD.

\section{Developmental regulators that traffic through PD}

Over the last two decades the importance of PD has been augmented by abundant and clear evidence that endogenous plant macromolecules use PD to move from cell to cell to influence development. Table 1 highlights two critical developmental regulators of cell identity, transcription factors, and small interfering RNAs (siRNAs), that traffic via PD. Most noncell-autonomous transcription factors are members of the KNOTTED1 homeobox (KNOX) or MADS domain families of proteins (reviewed in Jackson 2005). That multiple members of a given protein family traffic intercellularly from one tissue layer to another underscores such movement as essential for function. Movement of transcription factors is tightly regulated as most move only one to a few cells beyond their initial expression site (Nakajima et al. 2001; Kim et al. 2002b; Kim et al. 2003). The position of a plant cell is the key factor governing its developmental fate (van den Berg et al. 1995, 1997). Therefore the movement of transcription factors is a mechanism for conveying positional information to neighboring cells to reinforce other signals directing a differentiation process (Mezitt and Lucas 1996; Sessions et al. 2000). While numerous transcription factors move cell to cell via PD, none have been observed at sufficient microscopic resolution to reveal whether or not they accumulate to form PD puncta. However, KN1 dilates PD (Lucas et al. 1995), and this result implies a specific active interaction/targeting to PD.

Different types of siRNAs move cell to cell and act to silence endogenous and exogenous homologous sequences. Remnants from transposon inverted repeat sequences (IR) form a large fraction of eukaryotic genes, and such IRs are targets of endogenous gene silencing; we now design RNAinterference (RNAi) experiments that mimic this endogenous gene silencing strategy (Dunoyer et al. 2010b). Gene silencing is also critical to silence exogenous RNAs such as plant viruses (Mlotshwa et al. 2008). siRNAs likely move through PD as double-stranded RNA molecules and to date no proteins are known to associate with the siRNA complex (Dunoyer et al. 2010a). MicroRNAs (miRNAs) are regulators of developmentally important transcription factors and most act cell autonomously (reviewed in Voinnet 2009). However, new evidence suggests two miRNAs, miR165/6, move across cell files to regulate root development (Carlsbecker et al. 2010) discussed further below. Finally, some trans-acting silencing (tasi) RNAs move from the upper leaf surface to the lower leaf surface to signal auxin-mediated lower leaf development (Chitwood et al. 2009). Local PD-mediated and systemic movement of small ( 17-27 nucleotides) RNAs is a rapidly advancing new area of research in plant developmental biology.

PD aperture is regulated during development

PD were first described as channels allowing the passive movement of water and small solutes between cells (reviewed in Roberts and Oparka 2003). The advent of modern cell biological techniques combined with probes derived from plant viruses and membrane-impermeant fluorescent molecules of various sizes revealed the dynamic nature of PD. The upper limit of the size of molecules transported by PD is called the size exclusion limit (SEL), and SEL reflects PD aperture. Changes in PD aperture/SEL occur during all stages of plant growth and development, most often studied in tobacco leaves of different ages (Oparka et al. 1999 and references therein). However, it is important to note that within mature or immature tobacco 
Table 1 Developmental regulators of cell identity, transcription factors, and small interfering RNAs

\begin{tabular}{|c|c|c|c|c|}
\hline Molecule & Description & Function & Tissue & References \\
\hline \multicolumn{5}{|c|}{ Transcription factors } \\
\hline KN1 & KNOX family & Cell division and cell fate & Maize SAM & Lucas et al. 1995 \\
\hline GLO/DEF & $\begin{array}{l}\text { MADS domain family } \\
\text { and B type }\end{array}$ & Petal and stamen identity & $\begin{array}{l}\text { Antirrhinum } \\
\text { inflorescence } \\
\text { meristem }\end{array}$ & Perbal et al. 1996 \\
\hline LFY & Unique and plant specific & Floral meristem identity & $\begin{array}{l}\text { Arabidopsis } \\
\text { inflorescence }\end{array}$ & Sessions et al. 2000 \\
\hline STM & KNOX family & $\begin{array}{l}\text { SAM initiation and } \\
\text { maintenance }\end{array}$ & Arabidopsis SAM & Kim et al. 2003 \\
\hline KNAT1/BP & KNOX family & $\begin{array}{l}\text { SAM initiation and } \\
\text { maintenance; inflorescence } \\
\text { cell fate }\end{array}$ & $\begin{array}{l}\text { Arabidopsis } \\
\text { meristem }\end{array}$ & Kim et al. 2003 \\
\hline SHR & GRAS family & $\begin{array}{l}\text { Cell division and endodermis } \\
\text { specification }\end{array}$ & Arabidopsis root & $\begin{array}{l}\text { Helariutta et al. 2000; } \\
\text { Nakajima et al. } 2001\end{array}$ \\
\hline $\mathrm{CPC}$ & MYB family & Root hair development & Arabidopsis root & Wada et al. 2002 \\
\hline $\mathrm{AG}$ & $\begin{array}{l}\text { MADS domain family } \\
\text { and } C \text { type }\end{array}$ & $\begin{array}{l}\text { Cell division and cell fate; } \\
\text { flower development }\end{array}$ & $\begin{array}{l}\text { Arabidopsis } \\
\text { inflorescence }\end{array}$ & Urbanus et al. 2010 \\
\hline \multicolumn{5}{|l|}{ RNAs } \\
\hline $\begin{array}{l}\text { Endogenous } \\
\text { siRNAs }\end{array}$ & $\begin{array}{l}\text { 21-24 nt RNA from transcripts } \\
\text { with stem-loop structures }\end{array}$ & $\begin{array}{l}\text { Silence endogenous IR } \\
\text { sequences }\end{array}$ & All tissues & $\begin{array}{l}\text { Tretter et al. 2008; Dunoyer et al. } \\
\text { 2010a, b; Molnar et al. } 2010\end{array}$ \\
\hline tasiRNA & $\begin{array}{l}20-24 \mathrm{nt} \text { RNA, form by miRNA } \\
\text { activity, and siRNA machinery }\end{array}$ & Leaf development & Leaf & $\begin{array}{l}\text { Tretter et al. 2008; } \\
\quad \text { Chitwood et al. } 2009\end{array}$ \\
\hline miRNAs & $\begin{array}{l}\text { 20-24 nt RNA encoded by } M I R \\
\text { genes }\end{array}$ & $\begin{array}{l}\text { Cell fate and root } \\
\text { development }\end{array}$ & Root & Carlsbecker et al. 2010 \\
\hline
\end{tabular}

KN1 KNOTTED1, KNOX KNOTTED1 homeobox, SAM shoot apical meristem, GLO GLOBOSA, DEF DEFICIENS, LFY LEAFY, STM SHOOTMERISTEMLESS, KNAT1 KNOTTED1-like homeobox protein 1, BP BREVIPEDICELLUS, SHR SHORTROOT, $C P C$ CAPRICE, $A G$ AGAMOUS, siRNAs small interfering RNAs, tasi transacting siRNAs, miRNAs microRNAs

leaves individual cells may have PD with different states of aperture/SEL such as closed, open, or dilated, and the frequency of the different states of PD aperture varies in young versus older tissues (Crawford and Zambryski 2001). Remarkably, viral MPs can overcome reduced aperture even in mature leaves; $2 \mathrm{X}-\mathrm{GFP}(54 \mathrm{kDa})$ normally moves out of only $2 \%$ of the cells expressing it in mature source leaves, but TMV-MP fused to GFP (58 kDa) moves out of $52 \%$ of such cells (Crawford and Zambryski 2001). These data suggest that PD aperture is dynamic and not fixed.

As already mentioned, one important function of the dynamic regulation of PD aperture/SEL is the establishment of symplasmic fields, cytoplasmically coupled cells that execute a common developmental program. Symplasmic fields occur in the shoot apex (Rinne and van der Schoot 1998; Gisel et al. 1999; Gisel et al. 2002), root epidermis (Duckett et al. 1994), different regions of developing embryos (Kim et al. 2005a), seeds (Stadler et al. 2005), and the tissues surrounding the male and female gametophytes (Imlau et al. 1999). PD within a field will likely have similar SEL but reduced PD aperture at the borders likely modulates the passage of morphogens, allowing the establishment of morphogenetic gradients required for tissue development. Sometimes symplastic isolation is fundamental to the development of a particular cell type including root hairs (Duckett et al. 1994), stomatal guard cells (Palevitz and Hepler 1985), cotton fiber cells (Ruan et al. 2001), pollen mother cells that become isolated from the surrounding tapetum during their development (Regan and Moffatt 1990), and the female megaspore that becomes isolated from the surrounding cells of the ovule (Werner et al. 2010).

The viral connection: confusing primary and secondary PD

TMV-MP localizes to the central cavities of complex branched PD in the source leaves of transgenic tobacco plants constitutively expressing MP but is absent from simple PD in the sink leaves of the same plants (Ding et al. 1992). These data have led to the idea that TMV-MP only localizes to complex branched PD with central cavities, called "secondary" PD by the authors, in mature tissues (Ding et al. 1992). Many reports have subsequently asserted that viral MPs preferentially localize to "secondary" plasmodesmata in mature tissues (e.g., Epel 1994; Hofius et al. 2001). Based on these earlier reports, TMV-MP was used to distinguish branched ("secondary") PD from primary PD (Roberts et al. 2001). 

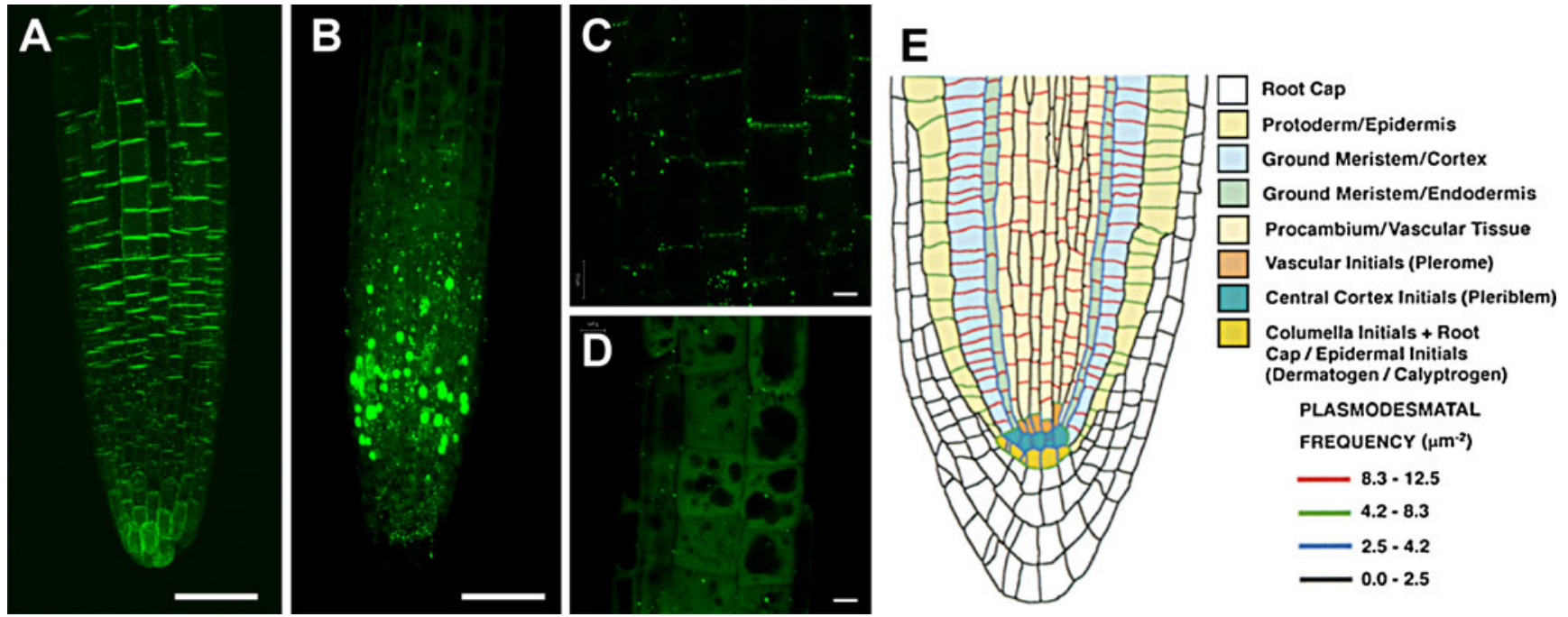

Fig. 2 Localization of viral movement proteins in Arabidopsis seedling root tips. TMV-MP-GFP constitutively expressed from the CaMV minimal 35S promoter in a 5-day-old transgenic Arabidopsis seedling root tips localizes predominantly to plasmodesmata in transverse cell walls (a) containing high densities of primary PD while PLRV MP17GFP expressed from the same promoter fails to localize to plasmodesmata in root tips (b). Images are projections of confocal stacks; scale bar represents $50 \mu \mathrm{m}$. Higher magnification confocal images of TMVMP-GFP (c) and PLRV MP17-GFP expression (d) in root tip epidermal cells reveal that TMV-MP-GFP is localized largely to punctae in transverse cell walls while PLRV MP17-GFP is retained in the cytoplasm and forms cytoplasmic aggregates. Scale bar represents $5 \mu \mathrm{m}$. e A graphic representation of the distribution and frequency of predominantly primary plasmodesmata in 1-week-old Arabidopsis root tips demonstrates that transverse cell walls in all cell layers of the root tip contain a higher density of plasmodesmata than longitudinal cell walls. e Adapted from Fig. 4 of Zhu et al. 1998b and used with kind permission from Springer: Protoplasma
However, it is critical to point out that TMV-MP is also capable of labeling simple PD (without branches or cavities) in immature cells. For example, TMV-MP fused to GFP (MP-GFP) significantly labels PD in cells from tobacco sink leaves (see Fig. 4 in Crawford and Zambryski 2001). Furthermore, TMV-MP-GFP extensively labels PD in embryos (Kim et al. 2005a), even though very few PD in embryonic tissues are secondary (Zhu et al. 1998b; BurchSmith and Zambryski 2010). Significantly, here we show that TMV-MP-GFP also labels the PD in transverse walls of cells in the differentiation zone just above the meristem of 1-week-old Arabidopsis seedling roots (Fig. 2a, c). These PD have been established as primary PD (Zhu et al. 1998a) and their distribution frequencies in the different cell files of seedling roots are illustrated in Fig. 2e. In support and in contrast the movement protein of, Potato leaf roll virus (PLRV) MP17, another MP that accumulates at complex PD in transgenic plants (Hofius et al. 2001), does not localize to PD in the same region of the developing root (Fig. 2b, d). Instead, PLRV MP17-GFP appears throughout the cytoplasm or in aggregates in the developing root (Fig. 2b, d).

While the literature claims that both TMV-MP and PLRV MP17 specifically label secondary PD, it is clear that TMVMP can also label primary PD in sink leaves, embryos, and young seedling roots. Thus, P30 and P17 have different and distinct PD-labeling specificities. Results that depend on the use of these probes to distinguish between PD of different origins should therefore be interpreted cautiously.

PD in different developing cells and tissues have differing transport capacities

PD of different origins and structures have different transport capabilities in different tissues. Arguably, strictly controlled differences in PD function have important roles in development to allow specific distribution of developmentally important molecules.

\section{Leaves}

Cell-to-cell movement has been studied most in tobacco leaves. In young leaves or in regions of maturing leaves that are still net photosynthetic sinks, greater than $90 \%$ of PD are simple (Oparka et al. 1999). As these cells become net exporters of photosynthate, simple PD become complex and sometimes elaborately branched PD (Ding et al. 1992; Itaya et al. 1998). While the origins of these latter PD were not determined, the authors refer to them as secondary. At least some of these complex PD may derive from modified (i.e., branched) primary PD. Nonetheless, it is clear that modified PD have different transport abilities from their simple progenitors.

Large soluble molecules like GFP move through PD by diffusion without specifically interacting with the PD, 
termed non-targeted movement (Crawford and Zambryski 2000). Cell-to-cell spread of GFP fusions revealed that the aperture/SEL of mesophyll PD of tobacco sink leaves is 47 to $54 \mathrm{kDa}$ (Oparka et al. 1999; Crawford and Zambryski 2001). However, source tissues showed a marked decrease in the ability to transport molecules of this size (Oparka et al. 1999; Crawford and Zambryski 2001). These results suggest that sink PD have a high basal aperture/SEL allowing diffusion-driven non-targeted transport of relatively large molecules, while source tissues have more restricted cell-to-cell movement.

In contrast to non-targeted movement of soluble GFP, TMV-MP-GFP targets to PD forming punctate patterns in the cell wall and remains associated with PD as it spreads to neighboring cells (Crawford and Zambryski 2000). When fluorescent membrane-impermeable dyes were microinjected into the cytoplasm of mature tobacco mesophyll cells, dyes of only approximately $500 \mathrm{Da}$ move freely from cell to cell (Wolf et al. 1989). However, in transgenic tobacco plants constitutively expressing TMV-MP, PD aperture/SEL increases to allow molecules of approximately $10 \mathrm{kDa}$ to move cell to cell following microinjection (Wolf et al. 1989). Thus in addition to mediating its own intercellular movement TMV-MP can "gate" PD, causing large increases in their apparent aperture/SEL and such "gating" stimulates movement of molecules (such as large fluorescent dextrans) in trans. PD gating was limited to fully expanded or mature leaves in TMV-MP transgenic tobacco when studied by microinjection assays (Deom et al. 1990). However, quantitative studies using biolistic bombardment to introduce TMV-MP for transient expression revealed that MP-GFP dramatically gates PD in source leaves $(52 \%$ of cells exhibited targeted movement of 58 $\mathrm{kDa}$ MP-GFP versus only $2 \%$ exhibited non-targeted movement of similarly sized $54-\mathrm{kDa} 2 \mathrm{X}$-GFP), and also causes significant gating in sink leaves $(76 \%$ of cells exhibited targeted movement of MP-GFP versus 30\% exhibited non-targeted movement of 2X-GFP) (Crawford and Zambryski 2001). Thus, TMV-MP causes a striking manipulation of aperture/SEL in complex PD of mature source leaf cells, but can also manipulate PD that already exhibit high aperture/SEL in immature sink leaf cells. That Deom et al. (1990) did not observe gating in immature tissues as in Crawford and Zambryski (2001) is likely due to several factors. Deom et al. (1990) used transgenic plants with long-term over-expression of TMV-MP that may lead to inactivation of TMV-MP by host factors, and fluorescent dextrans were added to cells by microinjection that may result in pressure induced PD closure (Oparka and Prior 1992). In contrast, Crawford and Zambryski (2001) studied TMV-MP activity following transient expression of DNA constructs introduced by biolistic bombardment at very low pressure. Note, early studies using biolistic bombardment at much higher pressure resulted in no diffusive non-targeted cell-to-cell movement of GFP (Itaya et al. 1997).

Independent dye coupling analyses in mature leaves, where the intercellular movement of an injected dye is monitored, reveal PD transport between specific cells: mesophyll to mesophyll, mesophyll to bundle-sheath cells, bundle-sheath cells to each other, but not bundle-sheath to phloem-parenchyma cells (Ding et al. 1992). This latter group of PD is truly secondary (Ehlers and Kollmann 2001), and is critical for unidirectional sugar transport (Volk et al. 1996). Thus secondary PD may have functions that are different from modified primary PD, despite their apparently identical structure.

\section{Trichomes}

Tobacco trichomes contain a linear array of five to eight cells that arise by cell division; thus, the PD connecting the cells are mainly primary, or secondary if they are formed post cell division. Trichome cells are connected by a gradient of simple (at the less mature tip cell) to complex, branched PD (in the more mature basal cell) (Waigmann et al. 1997). The basal aperture/SEL of PD between trichome cells is relatively large at $7 \mathrm{kDa}$ (determined by microinjection) and is not increased by TMV-MP (Waigmann and Zambryski 1995). Thus TMVMP cannot gate PD in all cell types.

A comprehensive study of PD at the tobacco leaf trichomeepidermal cell interface illuminates PD development during trichome maturation (Faulkner et al. 2008). The first PD formed at this interface are randomly distributed primary PD. As the surface area of the trichome-epidermal cell wall interface increases, there is an increase in the number of secondary PD due to the insertion of new PD directly adjacent to existing channels to produce twinned PD. These twinned PD likely represent one primary PD and one secondary PD and Faulkner et al. (2008) suggest they may fuse to give rise to branched PD with central cavities. Interestingly, there is unidirectional transport across this wall interface. The small ( $\sim 5 \mathrm{Da}$ ) fluorescent dye Lucifer yellow $\mathrm{CH}$ failed to enter epidermal cells when microinjected into the basal trichome cell, although it moved apically into other trichome cells (Christensen et al. 2009). However, if the dye was injected into the supporting epidermal cell, it entered the trichome cells. TMV-MP had no effect on this symplastic barrier, suggesting that the PD at this boundary are resistant to the gating effect of MP consistent with previous data (Waigmann and Zambryski 1995). Directionality of PD transport develops as leaves mature and PD undergo modification since PD in young single-celled trichomes, containing simple PD, allow transport into epidermal cells (Faulkner et al. 2008; Christensen et al. 2009). Another factor that may drive directional transport in mature trichomes is bulk solute transfer to tip cells for excretion. 


\section{Embryos}

The embryo has traditionally been viewed as a collection of immature tissues that establish the basic body plan of the adult plant. However, these developing tissues exhibit intricate regulation of their PD aperture/SEL (Kim et al. 2002a; Kim et al. 2005a, b; Kim and Zambryski 2005; Stadler et al. 2005) that is critical for normal Arabidopsis embryogenesis. For example, at the mid-torpedo stage PD aperture/SEL is significantly decreased (Kim et al. 2002a). Furthermore, the embryo contains distinct symplastic domains where single, double, and triple-sized GFP display differential movement into different regions of the embryo at different stages of embryogenesis (Kim et al. 2005a). Remarkably, these domains of PD-mediated transport correspond to the areas that will give rise to the basic tissues of the seedling established by clonal analyses, i.e., cotyledons, shoot apical meristem, hypocotyl, and root. Thus, the size of PD aperture and consequent transport capacity likely facilitates morphogenesis.

The identification of embryo-lethal mutants with altered patterns of movement of fluorescent tracers compared with wild type reinforces the importance of regulation of PD aperture/SEL in embryo development (Kim et al. 2002a). Two mutations, increased size exclusion limit (ise) 1 and 2, allow transport of fluorescent tracers beyond the time of the mid-torpedo transition when wild-type PD aperture/SEL is decreased. ISEI encodes a mitochondrial DEAD-box RNA helicase (Stonebloom et al. 2009), and ISE2 encodes a DEVH-type RNA helicase (Kobayashi et al. 2007). Benitez-Alfonso et al. (2009) mutagenized seedlings expressing GFP in phloem specific cells that lead to GFP movement cell to cell into surrounding tissues. They screened for mutants, called GFP-arrested trafficking1 (gat1) that exhibit reduced PD function by failing to unload GFP from the phloem.

Ultrastructural analyses examined the relationship between PD form and function in wild type versus ise 1 and ise 2 embryos (Burch-Smith and Zambryski 2010). Interestingly twinned and branched (Y- and H-shaped) PD occur in wildtype embryos of all stages representing $9 \%, 10 \%$, and $8 \%$ of PD in hypocotyls of early, mid, and late torpedo embryos (Burch-Smith and Zambryski 2010). Thus, in contrast to the widely held view that branched PD are synonymous with mature tissues, branched PD also occur in very young developing tissues. Notably ise 1 and ise 2 mutations result in a significant increase in the occurrence of twinned and branched PD in embryos (Burch-Smith and Zambryski 2010 ); isel embryos have $18 \%, 15 \%$, and $26 \%$ twinned and branched PD in hypocotyls of early, mid, and late torpedo stages, and ise 2 hypocotyls have $25 \%, 28 \%$, and $13 \%$ twinned and branched PD at the same stages. Interestingly there is a temporal pattern in the frequency of twinned and branched PD during embryogenesis; the hypocotyl contains more twinned and branched PD than the cotyledon in early and mid-torpedo embryos, and this pattern is reversed in late torpedo embryos. This pattern is conserved in the ise 2 mutant compared with wild type, but it is altered in the ise1 mutant. Thus, PD structure/function may regulate plant development or vice versa.

What is the origin of these twinned and branched PD in the embryo? RNA silencing experiments in sink leaves of Nicotiana benthamiana revealed that ISE1 and ISE2 act in pathways to control the formation of de novo secondary plasmodesmata; we were able to unambiguously assign PD as de novo secondary PD since we scored PD in the cell walls connecting the epidermal layer to the underlying mesophyll cells. Epidermal and mesophyll cells are not clonally related and divide independently of each other ((Marcotrigiano 2001) and references therein). Thus, we propose that the increased proportions of twinned and branched PD in isel and ise 2 mutant embryos are due to the upregulation of secondary PD formation. Secondary PD formed in ISEI- and ISE2-silenced leaves have significantly increased transport abilities compared with nonsilenced controls, as measured by TMV-MP-2XGFP movement in the horizontal and vertical planes representing PD-mediated movement between epidermal cells and from the epidermal layer to the underlying mesophyll cells. These data in silenced leaves are consistent with the increased transport of fluorescent tracers in ise1 and ise 2 mutant embryos (Burch-Smith and Zambryski 2010).

\section{$P D$ in the shoot apex}

PD connecting the L1 cells to underlying L2 cells in the shoot apical meristem (SAM) of dicotyledonous plants are unambiguously secondary as cells in the L1 layer almost exclusively divide anticlinally (Marcotrigiano 2001). Secondary PD connecting L1 to L2, such as those connecting the epidermis to the mesophyll layer of leaves can be simple or branched (Burch-Smith and Zambryski 2010). Secondary L1-L2 PD in the vegetative and inflorescence apical meristems facilitate movement of developmentally important transcription factors belonging to the KNOX (Jackson et al. 1994; Lucas et al. 1995) and the MADS families (Perbal et al. 1996) among others (Table 1). KNOTTED1 (KN1) was the first transcription factor shown to move intercellularly: KN1 RNA is expressed exclusively in L2 cells of maize meristems but $\mathrm{KN} 1$ protein is detected in both L1 and L2 (Lucas et al. 1995). Similarly, Antirrhinum DEFICIENS moves from L2 and L3 to the L1 (Perbal et al. 1996). When a GFP-KN1 fusion was expressed specifically in Arabidopsis leaf mesophyll cells (L2/L3) it was detected in the epidermis (L1), but it could not move in the opposite direction from epidermis to mesophyll (Kim et al. 2003). In contrast, when 
GFP-KN1 was expressed in the L1 of the SAM it moved to the L2/L3 layers. Thus, leaf L1-L2 secondary PD exhibit directionality of PD transport compared with L1-L2 secondary PD in the SAM (Kim et al. 2003). Free GFP and a GFP-viral MP fusion could move in both directions in the leaf, suggesting there is a specific mechanism regulating movement of the GFP-KN1 fusion and that PD are not generally closed in one direction.

PD and intercellular trafficking in the SAM are also regulated in response to environmental cues like seasonal day length changes that influence development. The frequency of PD throughout the Sinapis alba SAM undergoing the floral transition increases dramatically (Ormenese et al. 2000). While some PD may be primary due to cell division, Ormenese et al. (2000) suggest that the vast majority of these newly formed PD are secondary. Such secondary PD formation may be prompted by the transient increase in a floral stimulus (Ormenese et al. 2000), most likely cytokinin (Ormenese et al. 2006).

Detailed time course experiments indicate that PD transport is temporally regulated at the Arabidopsis SAM during development (Gisel et al. 1999; Gisel et al. 2002). There is little unloading of fluorescent tracers from the vascular system into young vegetative apices (plants with less than 12 visible leaves) but there is a dramatic increase of unloading into apices in older plants with greater than 12 visible leaves. (Loading is a term used to denote movement of nutrients, signal molecules and tracers into the vascular system from the surrounding cells of source leaves; the corollary is unloading which occurs when molecules move from the vascular system into the surrounding cells of sink tissues. For the experiments described tracer was loaded into the vascular system of leaf petioles, and then observed for unloading in the SAM sink.) Remarkably, unloading into the apex ceases for several days during the transition from vegetative to floral development in Arabidopsis (Fig. 3), and then resumes again once floral morphogenesis is underway (Gisel et al. 1999; Gisel et al. 2002). As the inflorescence meristem undergoes profound changes in architecture and gene expression to produce floral meristems (that will produce multiple organ types) versus the single type of organ (leaves) produced by the vegetative meristem, it may be advantageous to sequester the SAM during the establishment of reproductive development. Secondary PD formed during this time (Ormenese et al. 2000) may then facilitate highly regulated transport of micro- and macromolecular signaling molecules.

\section{$P D$ in vascular development}

The vascular cambium is a lateral meristem that gives rise to the secondary vascular tissues. An outstandingly detailed study utilized newly arising secondary xylem and phloem in

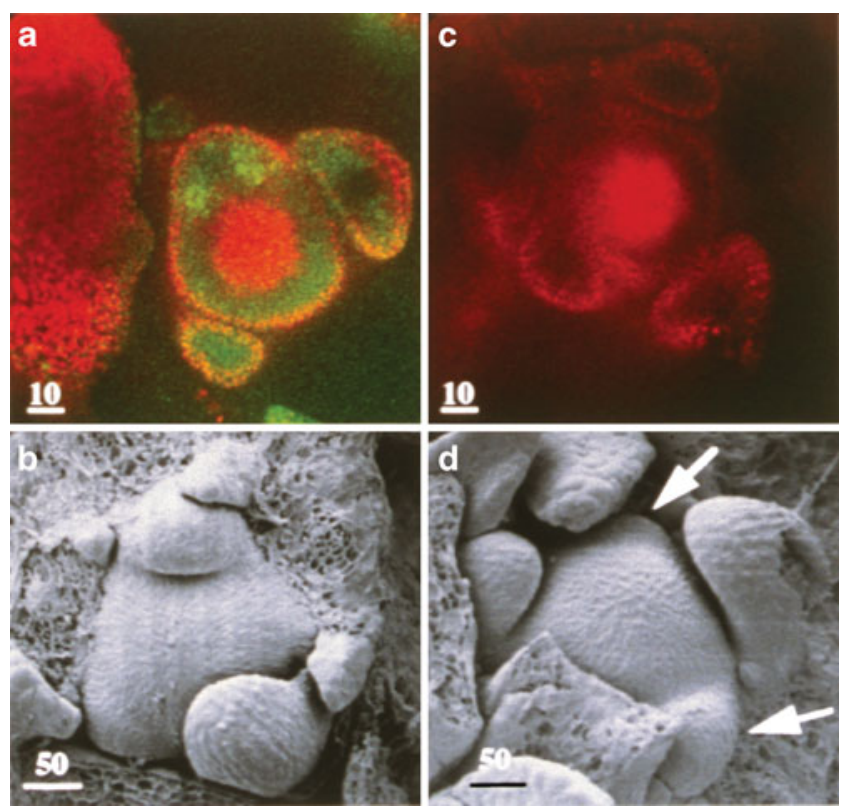

Fig. 3 Movement of symplastic tracers during the transition to floral development in Arabidopsis. Arabidopsis grown under short-day (SD) conditions were loaded through their leaf petioles with 8hydroxypyrene 1,3,6,-trisulfonic acid (HPTS), molecular mass 520 Da. Following 39 and 42 SDs, seedlings have 15 (a, b) and 19 leaves (c, d). a At $39 \mathrm{SD}$, plants are still vegetative and unload HPTS into their apices following transport via the vascular system from leaf petioles. The image is a confocal optical section and documents that HPTS does not unload in the center of the meristem (Gisel et al. 1999); however, HPTS does unload throughout leaf primordia seen around the circumference of the meristem. c At $42 \mathrm{SD}$, plants have initiated reproductive development and no longer unload HPTS into their apices. Vegetative versus reproductive apices are distinguished by the types of primordia they produce; leaf primordia are pointy versus floral primordia have a rounded shape (arrows in (d)). b Scanning electron micrograph (SEM) of a 39-SD-old apex reveals leaf primordia around the SAM. d SEM of a 42-SD apex reveals leaf primordia as well as newly arising floral primordia. Data were obtained as described in Gisel et al. 1999

the tomato stem to follow the production and function of PD during vascular development (Ehlers and van Bel 2010). PD development follows a strict chronological pattern that coincides with cell development, as also noted during embryo development (Burch-Smith and Zambryski 2010). Notably, most of the PD deployed in the cambial zone arise by modification of existing PD and extensive insertion of secondary PD (Ehlers and van Bel 2010). The authors describe four phases of PD development in tangential walls beginning with PD connecting the xylem and phloem initials and ending with PD connecting mature vascular cells. The radial patterning of cells during vascular development is dependent on increased branching of PD and insertion of secondary PD during cell expansion, and is concomitant with decreased intercellular transport (measured by injection of Lucifer Yellow and its movement in radial sections). In contrast, in transverse cell walls connecting cells of the same developmental stage, secondary PD are inserted in all 
developmental phases, not always coincident with cell expansion. Despite the large numbers of PD connecting longitudinal cell files, transport in cambial tissue is preferentially in the radial direction via PD between different cell types representing distinct developmental stages. This elaborate pattern of PD formation and modification likely serves to establish symplastic domains that are important for the differentiation of the secondary vasculature. There also are seasonal changes in PD trafficking in the cambium of poplar trees (Fuchs et al. 2010). Storage proteins that accumulate during the winter months are mobilized from vacuoles and storage bodies and are transported to growing buds during a 3 -week period in the spring coincident with reactivation of PD transport in cambial cells.

\section{$P D$ in roots}

Cell division patterns, cell expansion and PD distribution in the roots of Arabidopsis seedlings have been extensively characterized (Baum and Rost 1996; Rost et al. 1996; Zhu et al. 1998a; Zhu et al. 1998b; Benfey and Scheres 2000). The distribution of PD in developing Arabidopsis seedling roots as observed by Zhu et al. (1998a, b) is summarized in Fig. 2e. Since the cells of a developing root file are the product of successive transverse divisions, the PD in the transverse cell walls are predominantly primary, while those in the longitudinal walls connecting neighboring files of tissue are mainly secondary. There are more PD in the transverse walls than in longitudinal walls in young roots (Fig. 2e). This pattern is maintained through root development as cell expansion occurs, although the relative number of PD per $\mu \mathrm{m}^{2}$ in transverse walls decreases (Zhu et al. 1998b).

The cells of a particular vertical file or lineage share similar gene expression patterns (Rost and Bryant 1996; Brady et al. 2007) and are connected by numerous PD. After unloading from the phloem carboxyfluorescein moves preferentially through the transverse cell walls within a cell file of the root, consistent with the abundance of primary PD in those walls (Zhu et al. 1998a). Enhanced transport via PD may serve to reinforce both developmental programs within groups of cells and positional signaling from older to younger cells (van den Berg et al. 1995, 1997; Zhu et al. 1998a, b).

A striking example of the importance of PD-mediated intercellular traffic during development is how radial patterning is established in the root. The SHORTROOT (SHR) transcription factor moves from cell files in the central stele to the single layer of adjacent endodermal cells (Nakajima et al. 2001) (Table 1). Interestingly, SHR transport occurs via the secondary PD that connect the vertical walls in adjacent cell files, just as secondary PD are used for transcription factor movement in the apical meristem (see above). Note again that traffic through the secondary PD seems to be more tightly regulated than through primary $\mathrm{PD}$, since there is no dye coupling between cell files (Zhu et al. 1998a). SHR acts in the nucleus of the endodermis with its partner, the SCARECROW (SCR) transcription factor, to induce the expression of other transcription factors to promote root cell differentiation. Moreover, SHR and SCR induce the expression of specific miRNAs 165 and 166. miRNA165/166 then move cell to cell in the reverse direction to regulate the activity of the PHABULOSA (PHB) transcription factor by binding to PHB mRNA to promote root xylem development in a dose dependent manner (Carlsbecker et al. 2010). Lateral movement of miRNA 165/166 likely occurs via secondary PD.

How are PD apertures/SELs and transport regulated?

One of the outstanding questions about PD function is how aperture/SEL is regulated. The emerging picture suggests numerous cellular processes coordinate intercellular and intracellular signals that affect PD function/aperture.

\section{Callose}

In the dormant birch SAM, callose is deposited around PD neck regions leading to the isolation of individual cells and the breakdown of symplasmic fields (Rinne and van der Schoot 1998; Rinne et al. 2001). However, in the active birch SAM, callose is not associated with PD at the boundaries of active symplasmic fields (Rinne and van der Schoot 1998; Rinne et al. 2001). Also, callose deposition was not observed when PD transport was decreased in cells with clear differences in PD aperture/SEL that connect the differing cell types in the cambium and developing secondary vasculature of the tomato stems (Ehlers and van Bel 2010). Thus, while callose may be involved in long-term occlusion of PD during dormancy, it is not likely to be the main mechanism used for the transient regulation of PD during normal development. The importance of callose as a regulator of PD aperture is evidenced by the identification of a PD localized b-1,3-glucanase that regulates callose degradation (Levy et al. 2007). Reversibly glycosylated proteins (RGPs) can also localize to PD (Sagi et al. 2005), and they may regulate callose accumulation and PD function. In fact, RGP over-expression leads to decreased cellto-cell movement (Zavaliev et al. 2010) and silencing of RGP leads to enhanced PD-mediated transport (T.B.-S.,unpublished). The deposition of callose following wounding and during pathogen attack is an active area of investigation (see (Chen and Kim 2009) for review). Herein, Benitez-Alfonso, Jackson, and Maule review callose deposition at PD in response to changes in redox state.

\section{Cargo modification}

The best-studied PD interacting proteins are the viral MPs and several are phosphorylated in planta ((Lee and Lucas 
2001) and references therein). TMV-MP is phosphorylated at specific residues at its $\mathrm{C}$ terminus resulting in loss of its ability to move cell to cell and gate PD (Waigmann et al. 2000). TMV-MP is phosphorylated by a PD-resident kinase that is also active on a subset of other viral MPs and plant transcription factors including LEAFY (Lee et al. 2005). These results suggest an endogenous pathway uses phosphorylation to regulate cargo movement through PD.

\section{Energy and reactive oxygen species}

Inhibitor studies suggest that ATP-dependent mechanisms (apart from the previously mentioned phosphorylation) are important for regulating PD function. The general metabolic inhibitor sodium azide increases PD aperture/SEL while drastically reducing cellular ATP levels in a variety of tissues including wheat roots (Cleland et al. 1994), tobacco leaves (Christensen et al. 2009), and Setcreasea stamen hairs (Tucker 1993). ATP-dependent proteins such as actin may regulate PD transport in specific cell types. Application of the actin inhibitor latrunculin B caused an increase in PD aperture/SEL between leaf mesophyll cells (Ding et al. 1996) but not at the trichome-epidermis boundary (Christensen et al. 2009).

Recent evidence further implicates the general energy status of the cell, i.e., ATP production by mitochondria, in regulating PD transport. Embryo defective isel mutants increase PD transport and exhibit delayed development (Kim et al. 2002a). ISE1 encodes a nuclear encoded mitochondria localized RNA helicase that is likely essential for mitochondrial mRNA synthesis, and isel mutants are defective in the formation of mitochondrial electron transport gradients necessary for ATP production (Stonebloom et al. 2009). In addition, reactive oxygen species (ROS) likely act as critical signaling molecules between cellular energy status and PD development and/or function as ise 1 mutants produce increased levels of ROS (Stonebloom et al. 2009), and another Arabidopsis PD mutant, gat1, also produces increased levels of ROS (Benitez-Alfonso et al. 2009). GAT1 encodes a plastid thioredoxin and gat1 mutants increase callose production (Benitez-Alfonso et al. 2009). However, ise1 mutants have increased intercellular transport while gat 1 mutants have decreased intercellular trafficking (BenitezAlfonso et al. 2009; Stonebloom et al. 2009). Thus, the site of ROS production, mitochondria versus chloroplasts, likely differentially regulates cellular processes and associated specific targets that in turn affect PD function. In addition, the relative amount of ROS may differentially affect PD function as the levels of ROS produced in gatl are higher than those produced in isel.

Interestingly, thioredoxins, which are general regulators of the oxidative state of cells, can themselves move between cells (Ishiwatari et al. 1998; Meng et al. 2010). However, various thioredoxins differ in function and PD are implicated in the movement of only some thioredoxins in the vasculature (Ishiwatari et al. 1998). Further supporting the integral role of ROS in co-ordinating PD function, oxidative stress induced by anaerobic conditions increases PD aperture/SEL in wheat roots (Cleland et al. 1994).

\section{Perspectives}

Exciting advances in understanding PD form and function during development have been made recently. We look forward to progress in answering numerous outstanding questions. Now independent reports document the occurrence of twinned and branched PD in several immature tissues, leaves, roots, embryos, and vascular cambium. Does PD function differ in simple versus twinned or branched PD in such young tissues? Does PD function differ in primary versus secondary or twinned and branched $\mathrm{PD}$ in immature versus mature tissues? As PD-specific components are identified (Faulkner and Maule 2010) it will be interesting to determine their localization patterns during primary and secondary PD formation and PD modification throughout development. How do energy and ROS levels (reviewed by Benitez-Alfonso et al. 2010) signal PD formation and function during development? How widespread is miRNA movement? How do small RNAs interact with and move via PD? What factors control the movement of cell nonautonomous transcription factors that regulate development? How do secondary PD form? What are the roles of membranes and/or cell wall synthesis and degradation in secondary PD formation? What signals secondary PD to form? What signals PD to form branches or cavities? All these studies will benefit from the development of advanced imaging techniques as described by Oparka et al. (1992, 1999; Fitzgibbon et al. 2010). Finally, new data show that TMV-MP also labels PD in immature tissues, thus increasing its value as a versatile probe to study PD formation and function during all stages of developmental programming.

As we continue to make strides in elucidating and characterizing the critical roles of PD in plant development, it is important to adopt standard nomenclature for PD. We strongly encourage the use of the terms utilized by Ehlers and Kollmann (2001) to describe PD based on their origin and structure. The terms "primary" and "secondary" should be reserved for use when the origin of PD is unambiguously known. Simple and twinned PD are easiest to distinguish. Branched PD can be specified as Y- or Hshaped, or complex branched PD with central cavities. The latter degree of branching or cavity formation can be further specified, as they are clearly less complex in immature versus mature tissues. Moreover, as there are 2 types of secondary PD, it may be useful to adopt the terms "twinned 
secondary PD" and "de novo secondary PD". Clarity in PD nomenclature will ensure consistency and allow coherent and incisive discussions of existing and forthcoming data.

Acknowledgments We enthusiastically thank the Miller Institute for Basic Research in Science of UC Berkeley (TBS), the National Science Foundation Predoctoral Fellowship Program (SS), and the National Institutes of Health (GM 45244) (TBS, SS, MX, and PZ) for support.

Conflicts of interest The authors declare that they have no conflict of interest.

Open Access This article is distributed under the terms of the Creative Commons Attribution Noncommercial License which permits any noncommercial use, distribution, and reproduction in any medium, provided the original author(s) and source are credited.

\section{References}

Baum SF, Rost TL (1996) Root apical organization in Arabidops thaliana 1. Root cap and protoderm. Protoplasma 192:178-188

Benfey PN, Scheres B (2000) Root development. Curr Biol 10:R813R815

Benitez-Alfonso Y, Cilia M, San Roman A, Thomas C, Maule A, Hearn S, Jackson D (2009) Control of Arabidopsis meristem development by thioredoxin-dependent regulation of intercellular transport. Proc Natl Acad Sci USA 106:3615-3620

Benitez-Alfonso Y, Jackson D, Maule A (2010). Redox regulation of intercellular transport. Protoplasma (in press).

Brady SM, Orlando DA, Lee JY, Wang JY, Koch J, Dinneny JR, Mace D, Ohler U, Benfey PN (2007) A high-resolution root spatiotemporal map reveals dominant expression patterns. Science 318:801-806

Burch-Smith TM, Zambryski PC (2010) Loss of INCREASED SIZE EXCLUSION LIMIT (ISE)1 or ISE2 increases the formation of secondary plasmodesmata. Curr Biol 20:989-993

Carlsbecker A, Lee JY, Roberts CJ, Dettmer J, Lehesranta S, Zhou J, Lindgren $\mathrm{O}$, Moreno-Risueno MA, Vaten A, Thitamadee S, Campilho A, Sebastian J, Bowman JL, Helariutta Y, Benfey PN (2010) Cell signaling by microRNA165/6 directs gene-dose dependent root cell fate. Nature 465:316-321

Chen X-Y, Kim J-Y (2009) Callose synthesis in higher plants. Plant Signal Behav 4:489-492

Chitwood DH, Nogueira FTS, Howell MD, Montgomery TA, Carrington JC, Timmermans MCP (2009) Pattern formation via small RNA mobility. Genes Dev 23:549-554

Christensen NM, Faulkner C, Oparka K (2009) Evidence for unidirectional flow through plasmodesmata. Plant Physiol 150:96-104

Cilia ML, Jackson D (2004) Plasmodesmata form and function. Curr Opin Cell Biol 16:500-506

Cleland RE, Fujiwara T, Lucas WJ (1994) Plasmodesmal-mediated cell-to-cell transport in wheat roots is modulated by anaerobic stress. Protoplasma 178:81-85

Crawford KM, Zambryski PC (2000) Subcellular localization determines the availability of non-targeted proteins to plasmodesmatal transport. Curr Biol 10:1032-1040

Crawford KM, Zambryski PC (2001) Non-targeted and targeted protein movement through plasmodesmata in leaves in different developmental and physiological states. Plant Physiol 125:1802-1812

Deom CM, Schubert KR, Wolf S, Holt CA, Lucas WJ, Beachy RN (1990) Molecular characterization and biological function of the movement protein of tobacco mosaic virus in transgenic plants. Proc Natl Acad Sci USA 87:3284-3288

Ding B, Lucas WJ (1996) Secondary plasmodesmata:biogenesis, special functions, and evolution. BIOS Scientific Publishers, Oxford

Ding B, Haudenshield JS, Hull RJ, Wolf S, Beachy RN, Lucas WJ (1992) Secondary plasmodesmata are specific sites of localization of the tobacco mosaic virus movement protein in transgenic tobacco plants. Plant Cell 4:915-928

Ding B, Kwon MO, Warnberg L (1996) Evidence that actin filaments are involved in controlling the permeability of plasmodesmata in tobacco mesophyll. Plant J 10:157-164

Duckett CM, Oparka KJ, Prior DAM, Dolan L, Roberts K (1994) Dyecoupling in the root epidermis of Arabidopsis is progressively reduced during development. Development 120:3247-3255

Dunoyer P, Schott G, Himber C, Meyer D, Takeda A, Carrington JC, Voinnet O (2010a) Small RNA duplexes function as mobile silencing signals between plant cells. Science 328:912-916

Dunoyer P, Brosnan CA, Schott G, Wang Y, Jay F, Alioua A, Himber C, Voinnet O (2010b) An endogenous, systemic RNA1 pathway in plants. EMBO J 10:1699-1712

Ehlers K, Kollmann R (2001) Primary and secondary plasmodesmata: structure, origin, and functioning. Protoplasma 216:1-30

Ehlers K, van Bel AJ (2010) Dynamics of plasmodesmal connectivity in successive interfaces of the cambial zone. Planta 231:371-385

Epel BL (1994) Plasmodesmata: composition, structure and trafficking. Plant Mol Biol 26:1343-1356

Epel BL (2009) Plant viruses spread by diffusion on ER-associated movement-protein-rafts through plasmodesmata gated by viral induced host beta-1, 3-glucanases. Semin Cell Dev Biol 20:1074-1081

Faulkner C, Akman OE, Bell K, Jeffree C, Oparka K (2008) Peeking into pit fields: a multiple twinning model of secondary plasmodesmata formation in tobacco. Plant Cell 20:1504-1518

Faulkner C, Maule A (2010) Successes in the search for plasmodesmatal proteins. Protoplasma (in press)

Fitzgibbon J, Bell K, King E, Oparka K (2010) Super-resolution imaging of plasmodesmata using 3D-structured illumiination microscopy (3D-SIM). Plant Physiol 153:1453-1463

Fuchs M, van Bel AJ, Ehlers K (2010) Season-associated modifications in symplasmic organization of the cambium in Populus nigra. Ann Bot 105:375-387

Gisel A, Barella S, Hempel FD, Zambryski PC (1999) Temporal and spatial regulation of symplastic trafficking during development in Arabidopsis thaliana apices. Development 126:1879-1889

Gisel A, Hempel FD, Barella S, Zambryski P (2002) Leaf-to-shoot apex movement of symplastic tracer is restricted coincident with flowering in Arabidopsis. Proc Natl Acad Sci USA 99:1713-1717

Helariutta Y, Fukaki H, Wysocka-Diller J, Nakajima K, Jung J, Sena G, Hauser MT, Benfey PN (2000) The SHORT-ROOT gene controls radial patterning of the Arabidopsis root through radial signaling. Cell 101:555-567

Hofius D, Herbers K, Melzer M, Omid A, Tacke E, Wolf S, Sonnewald U (2001) Evidence for expression level-dependent modulation of carbohydrate status and viral resistance by the potato leafroll virus movement protein in transgenic tobacco plants. Plant J 28:529-543

Imlau A, Truernit E, Sauer N (1999) Cell-to-cell and long-distance trafficking of the green fluorescent protein in the phloem and symplastic unloading of the protein into sink tissues. Plant Cell 11:309-322

Ishiwatari Y, Fujiwara T, McFarland KC, Nemoto K, Hayashi H, Chino M, Lucas WJ (1998) Rice phloem thioredoxin h has the capacity to mediate its own cell-to-cell transport through plasmodesmata. Planta 205:12-22

Itaya A, Hickman H, Bao Y, Nelson R, Ding B (1997) Cell-to-cell trafficking of cucumber mosaic virus movement protein: green 
fluorescent protein fusion produced by biolistic gene bombardment in tobacco. Plant J 12:1223-1230

Itaya A, Woo YM, Masuta C, Bao Y, Nelson RS, Ding B (1998) Developmental regulation of intercellular protein trafficking through plasmodesmata in tobacco leaf epidermis. Plant Physiol 118:373-385

Jackson D, Veit B, Hake S (1994) Expression of maize KNOTTED1 related homeobox genes in the shoot apical meristem predicts patterns of morphogenesis in the vegetative shoot. Development 120:405-413

Jackson D (2005) Transcription factor movement through plasmodesmata. In: Oparka KJ (ed) "Plasmodesmata". Annual Plant Reviews 18:90-112

Kim I, Zambryski PC (2005) Cell-to-cell communication via plasmodesmata during Arabidopsis embryogenesis. Curr Opin Plant Biol 8:593-599

Kim I, Hempel FD, Sha K, Pfluger J, Zambryski PC (2002a) Identification of a developmental transition in plasmodesmatal function during embryogenesis in Arabidopsis thaliana. Development 129:1261-1272

Kim JY, Yuan Z, Cilia M, Khalfan-Jagani Z, Jackson D (2002b) Intercellular trafficking of a KNOTTED1 green fluorescent protein fusion in the leaf and shoot meristem of Arabidopsis. Proc Natl Acad Sci USA 99:4103-4108

Kim JY, Yuan Z, Jackson D (2003) Developmental regulation and significance of KNOX protein trafficking in Arabidopsis. Development 130:4351-4362

Kim I, Kobayashi K, Cho E, Zambryski PC (2005a) Subdomains for transport via plasmodesmata corresponding to the apical-basal axis are established during Arabidopsis embryogenesis. Proc Natl Acad Sci USA 102:11945-11950

Kim I, Cho E, Crawford K, Hempel FD, Zambryski PC (2005b) Cellto-cell movement of GFP during embryogenesis and early seedling development in Arabidopsis. Proc Natl Acad Sci USA 102:2227-2231

Kobayashi K, Otegui MS, Krishnakumar S, Mindrinos M, Zambryski P (2007) INCREASED SIZE EXCLUSION LIMIT 2 encodes a putative DEVH box RNA helicase involved in plasmodesmata function during Arabidopsis embryogenesis. Plant Cell 19:18851897

Lee JY, Lucas WJ (2001) Phosphorylation of viral movement proteins-regulation of cell-to-cell trafficking. Trends Microbiol 9:5-8

Lee JY, Taoka K, Yoo BC, Ben-Nissan G, Kim DJ, Lucas WJ (2005) Plasmodesmal-associated protein kinase in tobacco and Arabidopsis recognizes a subset of non-cell-autonomous proteins. Plant Cell 17:2817-2831

Levy A, Erlanger M, Rosenthal M, Epel BL (2007) A plasmodesmataassociated beta-1, 3-glucanase in Arabidopsis. Plant J 49:669-682

Lucas WJ, Lee JY (2004) Plasmodesmata as a supracellular control network in plants. Nat Rev Mol Cell Biol 5:712-726

Lucas WJ, Bouche-Pillon S, Jackson DP, Nguyen L, Baker L, Ding B, Hake S (1995) Selective trafficking of KNOTTED1 homeodomain protein and its mRNA through plasmodesmata. Science 270:1980-1983

Marcotrigiano M (2001) Genetic mosaics and the analysis of leaf development. Int J Plant Sci 162:513-525

McLean BG, Hempel FD, Zambryski PC (1997) Plant intercellular communication via plasmodesmata. Plant Cell 9:1043-1054

Meng L, Wong JH, Feldman LJ, Lemaux PG, Buchanan BB (2010) A membrane-associated thioredoxin required for plant growth moves from cell to cell, suggestive of a role in intercellular communication. Proc Natl Acad Sci USA 107:3900-3905

Mezitt LA, Lucas WJ (1996) Plasmodesmal cell-to-cell transport of proteins and nucleic acids. Plant Mol Biol 32:251-273

Mlotshwa S, Pruss GJ, Vance V (2008) Small RNAs in viral infection and host defense. Trends Plant Sci 13:375-382
Molnar A, Melnyk CW, Bassett A, Hardcastle TJ, Dunn R, Baulcombe DC (2010) Small silencing RNAs in plants are mobile and direct epigenetic modification in recipient cells. Science 328:872-875

Nakajima K, Sena G, Nawy T, Benfey PN (2001) Intercellular movement of the putative transcription factor SHR in root patterning. Nature 413:307-311

Oparka KJ (2005) Annual plant reviews. Plasmodesmata, vol 18. Blackwell, Oxford

Oparka K, Prior DAM (1992) Direct evidence for pressure gated closure of plasmodesmata. Plant J 2:741-750

Oparka KJ, Roberts AG, Boevink P, Santa Cruz S, Roberts I, Pradel KS, Imlau A, Kotlizky G, Sauer N, Epel B (1999) Simple, but not branched, plasmodesmata allow the nonspecific trafficking of proteins in developing tobacco leaves. Cell 97:743-754

Ormenese S, Havelange A, Deltour R, Bernier G (2000) The frequency of plasmodesmata increases early in the whole shoot apical meristem of Sinapis alba during floral transition. Planta 211:370-375

Ormenese S, Bernier G, Perilleux C (2006) Cytokinin application to the shoot apical meristem of Sinapis alba enhances secondary plasmodesmata formation. Planta 224:1481-1484

Palevitz BA, Hepler PK (1985) Changes in dye coupling of stomatal cells of Allium and Commelina demonstrated by microinjection of Lucifer yellow. Planta 164:473-479

Perbal MC, Haughn G, Saedler H, Schwarz-Sommer Z (1996) Noncell-autonomous function of the Antirrhinum floral homeotic proteins DEFICIENS and GLOBOSA is exerted by their polar cell-to-cell trafficking. Development 122:3433-3441

Regan SM, Moffatt BA (1990) Cytochemical analysis of pollen development in wild-type Arabidopsis and a male-sterile mutant. Plant Cell 2:877-889

Rinne PL, van der Schoot C (1998) Symplasmic fields in the tunica of the shoot apical meristem coordinate morphogenetic events. Development 125:1477-1485

Rinne PL, Kaikuranta PM, van der Schoot C (2001) The shoot apical meristem restores its symplasmic organization during chillinginduced release from dormancy. Plant J 26:249-264

Roberts AG, Oparka KJ (2003) Plasmodesmata and the control of symplastic transport. Plant Cell Environ 26:103-124

Roberts IM, Boevink P, Roberts AG, Sauer N, Reichel C, Oparka KJ (2001) Dynamic changes in the frequency and architecture of plasmodesmata during the sink-source transition in tobacco leaves. Protoplasma 218:31-44

Rost TL, Bryant JA (1996) Root organisation and gene expression patterns. J Exp Bot 47:1613-1628

Rost TL, Baum SF, Nichol S (1996) Root apical organization in Arabidopsis thaliana ecotype 'WS' and a commnet on root cap structure. Plant Soil 187:91-95

Ruan YL, Llewellyn DJ, Furbank RT (2001) The control of singlecelled cotton fiber elongation by developmentally reversible gating of plasmodesmata and coordinated expression of sucrose and $\mathrm{K}+$ transporters and expansin. Plant Cell 13:47-60

Sagi G, Katz A, Guenoune-Gelbart D, Epel BL (2005) Class 1 reversibly glycosylated polypeptides are plasmodesmal-associated proteins delivered to plasmodesmata via the golgi apparatus. Plant Cell 17:1788-1800

Sessions A, Yanofsky MF, Weigel D (2000) Cell-cell signaling and movement by the floral transcription factors LEAFY and APETALA1. Science 289:779-782

Stadler R, Lauterbach C, Sauer N (2005) Cell-to-cell movement of green fluorescent protein reveals post-phloem transport in the outer integument and identifies symplastic domains in Arabidopsis seeds and embryos. Plant Physiol 139:701-712

Stonebloom S, Burch-Smith T, Kim I, Meinke D, Mindrinos M, Zambryski P (2009) Loss of the plant DEAD-box protein ISE1 
leads to defective mitochondria and increased cell-to-cell transport via plasmodesmata. Proc Natl Acad Sci USA 106:17229-17234

Tretter EM, Alvarez JP, Eshed Y, Bowman JL (2008) Activity range of Arabidopsis small RNAs derived from different biogenesis pathways. Plant Physiol 147:58-62

Tucker EB (1993) Azide treatment enhances cell-to-cell diffusion in staminal hairs of Setcreasea purpurea. Protoplasma 174:45-49

Urbanus SL, Martinelli AP, Peter Dinh QD, Aizza LC, Dornelas MC, Angenent GC, Immink RG (2010) Intercellular transport of epidermis-expressed MADS domain transcription factors and their effect on plant morphology and floral transition. Plant J 63:60-72

van den Berg C, Willemsen V, Hage W, Weisbeck P, Scheres B (1995) Cell fate in the Arabidopsis root meristem determined by directional signaling. Nature 378:62-65

van den Berg C, Willemsen V, Hendricks G, Weisbeck P, Scheres B (1997) Short range control of cell differentiation in the Arabidopsis root meristem. Nature 390:287-289

Voinnet O (2009) Origin, biogenesis, and activity of plant microRNAs. Cell 136:669-687

Volk GM, Turgeon R, Beebe DU (1996) Secondary plasmodesmata formation in the minor-vein phloem of Cucumis pepo L. Planta 199:425-432

Wada T, Kurata T, Tominaga R, Koshino-Kimura Y, Tachibana T, Goto K, Marks MD, Shimura Y, Okada K (2002) Role of a positive regulator of root hair development, CAPRICE, in Arabidopsis root epidermal cell differentiation. Development 129:5409-5419

Waigmann E, Zambryski P (1995) Tobacco mosaic virus movement protein-mediated protein transport between trichome cells. Plant Cell 7:2069-2079
Waigmann E, Turner A, Peart J, Roberts K, Zambryski P (1997) Ultrastructural analysis of leaf trichome plasmodesmata reveals major differences from mesophyll plasmodesmata. Planta 203:75-84

Waigmann E, Chen MH, Bachmaier R, Ghoshroy S, Citovsky V (2000) Regulation of plasmodesmal transport by phosphorylation of tobacco mosaic virus cell-to-cell movement protein. EMBO J 19:4875-4884

Werner D, Gerlitz N, and Stadler R (2010) A dual switch in phloem unloading during ovule development in Arabidopsis. Protoplasma (in press)

Wolf S, Deom CM, Beachy RN, Lucas WJ (1989) Movement protein of tobacco mosaic virus modifies plasmodesmatal size exclusion limit. Science 246:377-379

Wu X, Dinneny JR, Crawford KM, Rhee Y, Citovsky V, Zambryski PC, Weigel D (2003) Modes of intercellular transcription factor movement in the Arabidopsis apex. Development 130:37353745

Zavaliev R, Sagi G, Gera A, Epel BL (2010) The constitutive expression of Arabidopsis plasmodesmal-associated class 1 reversibly glycosylated polypeptide impairs plant development and virus spread. J Exp Bot 61:131-142

Zhu T, Lucas WJ, Rost TL (1998a) Directional cell-to-cell communication in the Arabidopsis root apical meristem-I. An ultrastructural and functional analysis. Protoplasma 203:35-47

Zhu T, O'Quinn RL, Lucas WJ, Rost TL (1998b) Directional cellto-cell communication in the Arabidopsis root apical meristem II. Dynamics of plasmodesmatal formation. Protoplasma 204:84-93 